\title{
Note sul contributo di Jean-Luc Marion intorno al concetto di philosophia prima fra Descartes e la Scolastica*
}

Riassunto: Questo articolo ha per oggetto l'indagine che Jean-Luc Marion ha consacrato nei suoi numerosi scritti e, in particolare, in Sur le prisme métaphysique de Descartes (1986), alla concezione cartesiana della metafisica in rapporto alla scolastica. Esso si propone di mostrare che le ricerche di Marion costituiscono uno dei maggiori contributi della letteratura critica cartesiana allo studio dei rapporti fra Descartes e la Scolastica. In particolare, esso discute e riconsidera la tesi di Marion secondo cui la scelta, da parte di Descartes, del termine di philosophia prima per intitolare le sue Meditationes sarebbe operata a detrimento del termine di metaphysica.

Parole chiave: Cartesio. Jean-Luc Marion. Scolastica. Metaphysica. Philosophia prima.

\section{Notas sobre a contribuição de Jean-Luc Marion acerca do estudo do conceito de philosophia prima em Descartes e na Escolástica}

* $\quad$ AT = René Descartes, Euvres, éd. par Ch. Adam et P. Tannery, nouv. présent. par J. Beaude, P. Costabel, A. Gabbey et B. Rochot, 11 vols., Paris, Vrin, 19641974 ; B = René Descartes, Tutte le lettere 1619-1650 (2005), a cura di G. Belgioioso, con la collaborazione di I. Agostini, F. Marrone, F. A. Meschini, M. Savini e di J.-R. Armogathe, Bompiani, Milano, 2009; B Op I = René Descartes. Opere 1637-1649, a cura di G. Belgioioso, con la collaborazione di I. Agostini, F. Marrone, M. Savini, Bompiani, Milano, 2009; DM = F. Suárez, Disputationes metaphysicae, in Opera Omnia, 28 voll., Parisiis, apud Ludovicum Vivès, 1856-1878, voll. XXV-XXVI; Marion 1986 = Sur le prisme métaphysique de Descartes. Constitution et limites de l'onto-théo-logie dans la pensèe cartésienne, Paris, PUF, 1986.

** Professor de Filosofia da Universidade do Salento (Lecce). E-mail: igor.agostini@unisalento.it 
Resumo: Este artigo tem por objeto a indagação que Jean-Luc Marion consagrou nos seus numerosos escritos e, em particular, em Sur le prisme métaphysique de Descartes (1986), à concepção cartesiana da Metafísica em relação à Escolástica. Propõem-se mostrar que as pesquisas de Marion constituem uma das maiores contribuições da literatura crítica cartesiana ao estudo das relações entre Descartes e a Escolástica. Em particular, o artigo discute e reconsidera a tese de Marion segundo a qual a escolha, da parte de Descartes, do termo philosophia prima para intitular as suas Meditationes teria sido feita em detrimento do termo metaphysica.

Palavras-chave: Descartes. Jean-Luc Marion. Escolástica. Metaphysica. Philosophia prima.

\title{
Notes on Jean-Luc Marion's contribution on the study of the notion of prima philosophia in Descartes and the scholasticism
}

\begin{abstract}
This article addresses the enquiry devoted by Jean-Luc Marion in his many works and, in particular, in Sur le prisme métaphysique de Descartes (1986), to Descartes's conception of Metaphysics considered in connection to scholasticism. The aim is to demonstrate that Marion's researches constitue in Cartesian scholarship one of the main contributions to the study of the relationships between Descartes and Scholasticism. At the same time, the article discusses and reconsiders Marion's claim that Descartes's choice of the term philosophia prima for the title if his Meditationes would go to the detriment of the term metaphysica.
\end{abstract}

Keywords: Descartes. Jean-Luc Marion. scholasticism. Metaphysica. Philosophia prima.

I.

Le principali opere interpretative consacrate da Jean-Luc Marion a Descartes, ristampate a più riprese e in alcuni casi tradotte in più lingue, sono ben note: la fondamentale trilogia su L'ontologie grise (1975, II ed. rev. 1981), La théologie blanche (1981, II ed. rev. 1986) et Le prisme métaphysique (1986), un cospicuo numero di articoli di grande densità teorica, per lo più raccolto nei due volumi delle Questions cartésiennes 
(1991 e 1996) e, infine, l'ultima monografia su La pensée passive chez Descartes (2013) ${ }^{1}$.

Questi lavori offrono un contributo interpretativo allo studio di Descartes che, con ogni probabilità, è il maggiore dell'ultimo cinquantennio, dopo la grande generazione degli Alquié, Gouhier, Gueroult, Laporte. Non si tratta di un'opinione personale, ma di un dato oggettivo, attestato dalla larga diffusione delle tesi di Marion, che in Francia, e non solo, costituiscono spesso il punto di partenza di numerosi studi che si propongono in molti casi proprio di ridiscutere le stesse tesi interpretative marioniane.

Anzitutto due, sono gli aspetti del contributo di Marion che hanno suscitato l'attenzione degli studiosi. In primo luogo, la sua lettura interna dei testi cartesiani, ossia il suo apporto sul piano esegetico di questioni fondamentali quali la teoria della creazione delle verità eterne, il concetto cartesiano di metafisica, l'io, Dio, qui solo menzionate che alcune tematiche di particolare interesse. In secondo luogo, l'intenso confronto con la lettura di grandi filosofi quali, soprattutto, Husserl ed Heidegger: basti pensare, su quest'ultimo punto, al tentativo di lettura di Descartes alla luce del paradigma onto-teologico della metafisica realizzato nel Prisme e già abbozzato nella Théologie blanche, ma anche i contributi de La pensée passive verso l'individuazione dell'emergenza della chair rispetto al corpo fisico.

C'è però un altro aspetto della lettura di Marion, peraltro strettamente legato a quello interpretativo (che su di esso si appoggia), a mio parere, altrettanto importante, ossia il suo contributo ai rapporti fra Descartes e la Scolastica: credo si possa infatti affermare che in tale direzione il contributo di Marion sia senz'altro, dopo quello di Etienne Gilson, uno

Sur la théologie blanche de Descartes. Analogie, création des vérités éternelles et fondement (1981), PUF, Paris, 1991²; Descartes et l'onto-théologie, in «Bulletin de la Société française de philosophie», LXXVI (1982), pp. 117-171; Marion 1986; Questions cartésiennes. Méthode et métaphysique, Paris, PUF, 1991; Questions cartésiennes II. Sur l'ego et sur Dieu, PUF, Paris, 1996; Sur la pensée passive de Descartes, Paris, PUF, 2013. 
dei maggiori del secolo scorso, insieme a quello di autori quali Pierre Garin, Timothy J. Cronin e, Norman J. Wells.

Il suo fondamentale apporto si estende difatti, limitandomi al nucleo essenziale, a quattro questioni: la questione dell'analogia; la questione della metafisica; la questione dei nomi divini; la questione della sostanza. Si tratta di tematiche capitali che in questa sede, per ragioni di spazio, non mi è possibile ripercorrere a dovere. Mi soffermerò, infatti, solo su una di esse, sia per la sua importanza, sia perché, forse, a differenza delle altre, ha suscitato meno attenzione all'interno del dibattito storiografico successivo.

Mi riferisco, in particolare, alla questione della metafisica, oggetto, peraltro, del primo capitolo de Le prisme métaphysique de Descartes.

\section{II.}

Il punto di partenza dell'indagine di Marion sul concetto cartesiano di metafisica è costituito dalla stessa accezione del termine che si ritrova, in Descartes, sin dalle lettere del 1630 a Mersenne sulle verità eterne:

Pensai-je avoir trouvé comment on peut démontrer les vérités métaphysiques, d'une façon qui est plus évidente que les démonstrations de Géométrie ${ }^{2}$.

Poiché la metafisica è qui intesa come scienza che sorpassa le altre scienze («Dieu est une cause dont la puissance surpasse les bornes de l'entendement humain $»^{3}$ ), Marion vi ritrova il concetto aristotelico di philosophia prima, o meglio, l'etimologia della philosophia prima quale scienza che si eleva, in quanto prima, al di sopra delle altre scienze teoriche.

Si tratta di un'etimologia largamente diffusa nella scolastica degli anni di Descartes, come lo stesso Marion dimostra attraverso il reperimento di numerosi testi:

AT I 144; B 30, p. 144.

AT I 150; B 31, p. 150. 
[...] Hinc factum est; ut scientia ipsa, quæ his libris traditur, simplici nomine appellari soleat a Recentioribus Metaphysica, quasi postnaturalium aut Supranaturalium scientia, cum ab Aristotele sæpe appellatur prima philosophia ${ }^{4}$.

[...] Ex quo etiam metaphysica nominata est; quod nomen non tam $\mathrm{ab}$ Aristotele, quam ab ejus interpretatibus habuit; sumptum vero est ex inscriptione quam Aristoteles suis Metaphysicæ libris præscripsit,

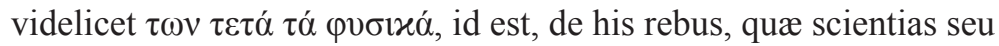
res naturales consequuntur ${ }^{5}$.

[...] hanc scientiam transphysicam seu postphysicam vocavit [sc. Aristotele] $]^{6}$.

[...] quia Physicam ordine doctrinæ sequitur, sive quia illam prætergreditur?

[...] Tandem appellatur Metaphysica, id est post-naturalis scientia, seu disciplina rerum res naturales consequentium ${ }^{8}$.

Difatti Descartes, in linea con questa tradizione, scrive: «La métaphysique se définit et se constitue par l'acte même de transgresser toute autre science» ${ }^{9}$. Questo non solo nel 1630, ma anche nel 1647, nella Lettre-Préface dei Principes de la philosophie, quando la metafisica

4 P. Fonseca, Commentarii in libros metaphysicorum Aristotelis Stagiritae, 4 voll., Coloniæ, Sumptibus Lazari Zetzneri, 1615-1619, rist. anast. : Hildesheim, G. Olms, 1964 (= Metaphysica), Proem., 8, vol. I p. 35, cit. in Marion 1986, p. 24, n. 20.

5 DM, I, 1, Proem., ed. Vivès XXV 2b, cit. in Marion 1986, pp. 24-25, n. 21.

6 DM, I, 4, 13, ed. Leon. XXV 29a, cit. in Marion 1986, pp. 24-25, n. 21.

7 Eustachius a S. Paulo, Summa philosophiae quadripartita, Parigi, apud Carolum Chastellain 1609 (= Summa philosophice), Metaphysica, Praefatio, 1, p. 1., cit. in Marion 1986, p. 25 nota 22 .

8 Abra de Raconis, Summa totius philosophice : id est, logica, ethica, physica, metaphysica et horum omnium Compendium, Coloniæ Agrippinæ, ex officina Choliniana, suptibus Petri Cholini, 1629 (= Summa totius philosophice), Metaphysica, Præfatio, p. 1., cit. in Marion 1986, p. 25, n. 22.

9 Marion 1986, p. 25. 
cartesiana ha ormai acquisito una fisionomia ben definita: «[...] la vraie Philosophie, dont la première partie est la Métaphysique» ${ }^{10}$.

Inoltre, il debito di Descartes nei confronti della tradizione scolastica si rileva, secondo Marion, anche in un secondo carattere che l'autore delle Meditationes attribuisce alla metafisica: l'astrazione dalle condizioni sensibili. La metafisica comincia difatti per Descartes allorché la mente si ritrae dai sensi, indirizzandosi verso le «res intelligibiles tantum, atque $\mathrm{ab}$ omni materia secretas ${ }^{11}$.

È, questo, un altro tratto tipico della metafisica secondo i maggiori autori dell'epoca di Descartes rilevato da Marion, ancora una volta, attraverso numerose citazioni tratte dai principali testi scolastici del tempo:

Tertia est abstractio rei ab omni materia, tam secundum rem, quam secundum rationem, quam abstractionem cernimus in Deo et intelligentiis $[\ldots]^{12}$.

Abstrahit enim hæc scientia de sensibilibus seu materialibus rebus (quæ physice dicuntur, quoniam in eis naturalis philosophia versatur) et res divinas et materia separatas et communes rationes entis, quæ absque materia existere possunt, contemplatur: et ideo metaphysica dicta est, quasi post physicam seu ultra physicam constituta ${ }^{13}$.

[...] metaphysicam esse scientiam quæ ens, in quantum ens, seu in quantum a materia abstrahit secundum esse, contemplatur ${ }^{14}$.

[...] ens abstractum a Deo et creaturis statuendum est adæquatum metaphysicæ objectum ${ }^{15}$.

$10 \quad$ AT IX-2 14; B Op I 2228.

11 AT VII 53, B Op I 750.

12 Pererius, De Communibus omnium rerum naturalium principiis et affectionibus (1576), Parisiis : apud Micaelem Sonnium, via Iacobea, sub scuto Basiliensi, 1579 (= De Communibus), I, 5, p. 16, ed. 1579, cit. in Marion 1986, p. 28, n. 26.

13 Suárez, DM, I, Proemium, ed. Vivès XXV 2a, in Marion 1986, p. 28, n. 26.

14 Suárez, DM, I, 3, 1, ed. Vivès, XXV 22b, in Marion 1986, p. 28, n. 26.

15 Abra de Raconis, Summa totius philosophice, Metaphysica, Præludium quartum, 2, prop. post., p. 4, in Marion 1986, p. 28, n. 26. 
Ex his colligi potest hæc Metaphysicæ definitio ut videlicet dicatur scientia theorica de ente reali per se et completo ab omni materia saltem secundum indifferentiam abstracto ${ }^{16}$.

Certamente, Descartes allarga in una direzione sconosciuta agli autori scolastici questa nozione di metafisica: essa non astrae solo dalle cose sensibili, ma si spinge sino ad abducere mentem a sensibus ${ }^{17}$. Ma questa estensione, lungi dall'attenuare la continuità della concezione cartesiana della metafisica con quella scolastica, la rinforza:

Les occurrences cartésiennes de 'métaphysique', quelque rares et éparses qu'elles soient, retrouvent donc une réelle cohérence sitôt qu'elles sont rapportées aux déterminations aristotéliciennes, mais aussi scolaires, admises par les contemporains de Descartes. Ainsi, dès 1630, Descartes reprend-il à son compte l'ambition d'Aristote, en E1, de constituer une science outrepassant la physique et les mathématiques; il reprend aussi l'abstraction comme l'acte privilégié pour transgresser les deux sciences secondes, et il en élargit la puissance par l'abductio mentis a sensibus ${ }^{18}$.

Questo dato non deve essere in alcun modo trascurato: basti pensare che, nell'Index scolastico-cartésien di Gilson, la voce metafisica costituisce solo una piccola sottovoce (solo quattro testi) della voce 'Fi-

16 Eustachius a S. Paulo, Summa philosophice, Metaphysica, Prcefatio, 2, p. 5, in Marion 1986, p. 29, n. 27.

17 Synopsis, B Op I 695 (AT VII 12, 11. 7-8); Meditationes, IV, B Op I 751 (AT VII 52, 11. 23-24); A Mersenne, 20 aprile 1637, B 104, p. 367 (AT I 350, 1. 29-351, 1. 2); A Vatier, 22 febbraio 1638, B 149, p. 549 (AT I 560, 11. 13-27). Cfr. anche Discours, IV, B Op I 67 (AT VII 37 11. 1-5) e, l'aggiunta: «ex eo est quod nunquam animum a sensibus abducant, et supra res corporeas attollant» in R. Descartes, Specimina Philosophiae seu: Dissertatio de Methodo recte regendae rationis, et veritatis in scientiis investigandae: Dioptrice, et Meteora. Ex gallico translata et ab auctore perlecta, variisque in locis emendata, Amstelodami, apud Ludovicum Elzevirium, 1644, rist. anast.: Lecce, Conte, 1998, a cura di J.-R. Armogathe e G. Belgioioso, p. 34.

18 Marion 1986, p. 33. 
losofia' ${ }^{19}$. Ma è proprio in tal modo che si genera la questione decisiva, secondo Marion: una volta dimostrato, cioè, che il termine ed il concetto di metafisica intervengono nel corpus cartesiano, occorre difatti chiarire quale sia il concetto propriamente cartesiano di metafisica:

Sur ce sol désormais assuré peut s'énoncer enfin la question décisive: puisque intervient bien «métaphysique» dans le corpus cartésien, comment s'y trouve proprement compris le concept de métaphysique? ${ }^{20}$

Il che significa misurare, precisamente «grâce a ces similitudes, les dissimilitudes qui provoquent les décisions proprement cartésiennes», ovverosia «s'interroger sur l'identité de la métaphysique instaurée en 1641 par les Meditationes» ${ }^{21}$. Ora, secondo Marion:

Or, c'est précisément ici que le déplacement s'accomplit le plus indiscutablement, puisque Descartes dénie expressement aux Meditationes le titre de metaphysicæ. L'originalité des Meditationes tient à ceci, qu'elles ne sont pas metaphysicæ' ${ }^{22}$.

Si tratta, sostiene Marion, di un fatto mascherato dalla traduzione francese delle Meditationes, che De Luynes rende appunto Méditations métaphysiques, ma anche, almeno in parte, da Descartes stesso, che, come avremo modo di ripetere più avanti, presenta a più riprese le Meditationes come «ma métaphysique». Tuttavia, è Descartes stesso che, per due volte, espone con nettezza i motivi che lo interdicono nel qualificare le Meditationes come «metaphysicæ». Questo, nelle

19 Cfr. E. Gilson, Index scolastico-cartésien. Seconde édition revue et augmentée seule autorisée par l'auteur (1913), Paris, Vrin, 1979, textes nn. 358-361. Nell'aggiornamento dell'opera di Gilson condotto nel Nouvel Index scolastico-cartésien, a cura del sottoscritto, con la collaborazione di S. Agostini, C. Catalano e I. Coluccia, la voce «metaphysica» tiene largamente conto della documentazione testuale offerta da Marion.

$20 \quad$ Marion 1986, p. 33.

21 Marion 1986, p. 34.

22 Marion 1986, p. 34. 
due lettere di invio a Mersenne delle Meditationes, datate entrambe 11 novembre 1640 :

J'ai envoyé dès hier ma Métaphysique à $\mathrm{M}$. de Zuylichem pour vous l'adresser; mais il ne l'enverra que dans huit jours, car je lui ai donné ce temps pour la voir. Je n'y ai point mis de titre, mais il me semble que le plus propre sera de mettre Renati Descartes Meditationes de prima Philosophia; car je ne traite point en particulier de Dieu et de l'Ame, mais en général de toutes les premières choses qu'on peut connaître en philosophant ${ }^{23}$.

Je vous envoie enfin mon écrit de Métaphysique, auquel je n'ai point mis de titre, afin de vous en faire le parrain, et vous laisser la puissance de le baptiser. Je crois qu'on le pourra nommer, ainsi que je vous ai écrit par ma précédente, Meditationes de prima Philosophia; car je n'y traite pas seulement de Dieu et de l'Ame, mais en général de toutes les premières choses qu'on peut connaître en philosophant par ordre ${ }^{24}$.

Secondo l'ipotesi di Marion, questi testi attesterebbero in maniera inequivocabile una decisione terminologica costante, da parte di Descartes, che è, a un tempo, a favore di philosophia prima, e a detrimento $d i$ metaphysica:

Il s'agit donc d'une decision nette et constante, au detriment de métaphysique/metaphysica, et en faveur de la philosophie premièrel prima philosophia, au sens strict de la filosof.... a prèth et suivant la nomenclature d'Aristote ${ }^{25}$.

Questa scelta lessicale induce una questione senza rispondere alla quale la scelta di Descartes, per quanto netta, resterebbe insignificante: perché una tale scelta, appunto, da parte di Descartes? Secondo l'ipotesi

23 AT III 235; B 284, p. 1324.

24 AT III 240; B 285, p. 1328.

25 Marion 1986, p. 35-36. 
di Marion, il fatto è che Dio e l'anima esauriscono, in Descartes, il campo della metafisica e, conseguentemente, si richiede una nuova disciplina che abbia per oggetto, essa sola, tutte le prime cose, quali esse siano:

Puisque Dieu et l'âme épuisent pour Descartes le champ de la métaphysique, l'élargissement de ce champ postule aussitôt une nouvelle discipline - la philosophie première, qui porte, elle seule, sur toutes les premières choses, quelles qu'elles soient et quelle qu'en soit la primauté26.

In questo modo:

[...] la philosophie première outrepasse la métaphysique en s'étendant à toute primauté; ainsi est-elle universelle en tant que première ${ }^{27}$.

La primauté in questione è quella nell'ordine della conoscenza. Il punto è capitale, in quanto determina una reinterpretazione della stessa primauté che viene staccata da ogni ambito ontologico (sia di Dio e dell'anima, sia dell'ente in generale) e collocata puramente sull'ordine della conoscenza: di conseguenza, la filosofia prima non desumerà la sua primauté da determinate regioni dell'ente supposte intrinsecamente prime (Dio, l'anima, le intelligenze separate), né dall'ente in quanto tale, poiché, una volta entrato in scena l'ordo cognoscendi, l'ente non entra più in gioco.

Una tale concezione della metafisica è ratificata dalla definizione della Lettre-Préface ai Principes:

[...] La vrai philosophie dont la première partie est la Métaphysique, qui contient les principes de la connaissance ${ }^{28}$.

26 Marion 1986, p. 37.

27 Marion 1986, p. 38.

28 AT IX-2 14; B Op I 2228. 
Definizione capitale, che, via Clauberg, giungerà sino a Wolff, e sarà assunta in proprio anche da Kant. Così, lungi dal rimettere in questione il primato della filosofia prima, la scelta terminologica di 'metafisica' la conferma, alla luce del nuovo concetto di primauté. In questo modo, «Descartes pense la métaphysique comme philosophie première, parce qu'il modifie de fond en comble l'essence de la primauté» ${ }^{29}$.

Per comprendere l'importanza di una tale decisione è necessario misurarne la portata concettuale e, a tal fine, rapportare la definizione cartesiana di filosofia alle definizioni precedenti, sia di metafisica, sia di filosofia prima.

È qui che l'analisi marioniana del background scolastico della filosofia di Descartes si fa più intensa. Il paragrafo 4 del capitolo I del Prisme contiene un contributo decisivo alla ricostruzione delle differenti definizioni di metafisica e di filosofia prima della scolastica dell'età moderna, del tutto assente nell'ambito della storiografia cartesiana, ivi compreso l'Index scolastico-cartésien di Gilson.

Dalle analisi di Marion risulta con evidenza che, nei contemporanei di Descartes, è largamente diffusa una tesi: la metaphysica ha per oggetto sia gli enti più nobili, sia l'ente in quanto ente. Le due scienze che in Aristotele sono distinte, o, comunque, non riunite sotto un titolo comune, ossia la philosofia prima che ha per oggetto gli enti più divini, e 'quella scienza' che ha per oggetto l'ente in quanto ente, sono senz'altro unificate sotto l'unico nome di 'metafisica'.

Così, in Suárez:

[...] Hæc scientia [...] tractat [...] de nobilissimis entibus et de universalissimis rationibus entis et maxime abstractis ${ }^{30}$.

Nell'affermare, così esplicitamente, l'unità specifica della metafisica, Suárez utilizza però, nondimeno, una distinzione terminologica:

29 Marion 1986, p. 43.

30 Suárez, DM, I, 4, 2, ed. Vivès XXV 26b, cit. in Marion 1986, p. 43, n. 42. 
Nihilominus asserendum est cum communi sententia, metaphysicam simpliciter esse unam scientiam specie. Hæc enim videtur clara mens Aristotelis in toto prooemio, seu c. 1 et 2, lib. I Metaph., ubi semper de hac scientia tamquam de una specie loquitur, eique tamquam uni et eidem attribuit nomina et attributa, quæ partim illi conveniunt, secundum quod versatur circa Deum et intelligentias; sic enim vocatur theologia, seu scientia divina, et prima philosophia; partim ut versatur circa ens in quantum ens, et prima attributa, et principia eius, qua ratione dicitur scientia universalis et metaphysica. Sapientia autem vocatur, quatenus hæc omnia complectitur, et prima principia, primasque rerum causas contemplatur ${ }^{31}$.

In breve, posta l'unità specifica della metafisica quale scienza, per Suárez la filosofia prima (in questo, sinonimo di teologia e scienza divina) indica la scienza che ha per oggetto Dio e le intelligenze; la metafisica (in questo, sinonimo di scienza universale) indica la scienza che ha per oggetto l'ente in quanto ente. È proprio qui che si situa la differenza capitale fra Descartes e Suárez. Infatti, se per Suárez la philosophia prima non concerne che Dio e le intelligenze, per Descartes è la metafisica ad avere per oggetto gli enti più nobili (Dio e l'anima), mentre la filosofia prima ha per oggetto le prime cose che possono conoscersi filosofando con ordine.

In tal modo, la metafisica, che per Suárez copre un dominio universale dell'ente (l'ente in quanto ente), per Descartes investe un dominio più particolare (Dio e l'anima); e la filosofia 'prima', che per Suárez copre un dominio particolare dell'ente (Dio e le intelligenze), per Descartes investe un dominio più in generale. In sostanza:

Tout se passe comme si Descartes ne reprenait le couple suárezien de prima philosophia/metaphysica que pour en inverser les caractéristiques: la première devient universelle et non théologique, la seconde devient particulière et théologique ${ }^{32}$.

31 Suárez, DM I, 3, 9, ed. Vivès XXV 24b.

32 Marion 1986, p. 46. 
Ad essere ridefinita, qui, è la primauté della filosofia prima, stabilita non dall'ordine dell'essere, ma dalla conoscenza e, insieme, dall'universalità. Un tale ribaltamento, secondo Marion, merita tanto più attenzione quanto più contraddice l'uso dei contemporanei di Descartes. Oltre che in Suárez, infatti, è largamente attestata nella gran parte degli autori scolastici dell'epoca anche l'identificazione dell'oggetto della filosofia prima agli enti più nobili e dell'oggetto della metafisica all'ente in quanto ente. Alcuni degli esempi addotti da Marion lo attestano in modo cristallino:

Dissentiunt de re propositia Philosophi; alii enim Deum, alii substantias separatas, alii substantiam in communi, alii ens finitum quod vocant prædicamentale, objectum esse Metaphysicæ contendunt: sed hi omnes non satis amplum illud assignant, ut patebit. [...] Est igitur longe verisimilior communis sententia: Objectum per se et adæquatum Metaphysicæ (de eo enim, non vero de objecto per accidens, aut partiali proposita quæstio intelligenda est) esse ens reale, per se, completum, commune Deo et rebus creatis [... $]^{33}$.

[...] hæc Metaphysicæ definitio, ut videlicet dicatur theoretica de ente reali per se et completo ab omni materia saltem secundum indifferentiam abstracto ${ }^{34}$.

$[\ldots]$ nomine primæ philosophiæ $[\ldots]$ cum de præstantissimis rebus, Deo nempe et angelis, verba faciat ${ }^{35}$.

La posizione di Descartes non è, tuttavia, senza antecedenti. C'è infatti un autore ${ }^{36}$ che aveva invece inteso la filosofia prima come

33 Eustachius a S. Paulo, Summa philosophice, Metaphysica, Præfatio, 2, p. 3, cit. in Marion 1986, p. 47, n. 46.

34 Eustachius a S. Paulo, Summa philosophice, Metaphysica, Præfatio, 2, p. 5, cit. in Marion 1986, p. 47, n. 46.

35 Abra de Raconis, Summa totius philosophice, Metaphysica, Præfatio, p. 1, cit. in Marion 1986, p. 46, n. 44.

36 La cui originalità era stata richiamata già da E. Vollrath, cui Marion 1986, p. 46, n. 9, si richiama esplicitamente: cfr. E. Vollrath, Die Gliederung der Metaphysik in eine 
universale: Benito Pereira, nel De communibus omnium rerum naturalium principiis et affectionibus libri quindecim (1576). Qui, infatti, senz'altro, Pereira sostiene che l'oggetto della filosofia prima sono le proprietà trascendentali ed universali, mentre l'oggetto della metafisica sono le intelligenze:

[...] Necesse est duas scientias distinctas inter se. Unam quæ agat de trascendentibus et universalissimis rebus. Alteram quæ de intelligentiis. Illa dicitur prima Philosophia et scientia universalis, hæc vocabitur proprie Metaphysica, Theologia, sapientia, divina scientia $[\ldots]^{37}$.

La tesi di una possibile influenza di Pereira su Descartes su questo punto è sostanziata dal fatto che, come dimostrato da Giovanni Crapulli, è noto che Descartes conoscesse lo stesso, poiché è da lui che desume il sintagma di Mathesis universalis ${ }^{38}$. Ma, al di là dell'effettiva influenza storica, Marion insiste sulla convergenza tematica: la primauté della filosofia prima resta definita non dalla nobiltà del suo oggetto, ma dalla sua universalità. Tuttavia, secondo Marion, la convergenza di Descartes con Pereira è solo molto parziale, puramente nominale. Per Pereira, difatti, la filosofia prima ha per oggetto le determinazioni trascendentali dell'ente, dunque, in ultima istanza, l'ente in quanto ente. La sua universalità, pertanto, dipende dall'universalità delle determinazioni dell'ente stesso. In questo modo, da un lato, Pereira anticipa la scienza che dopo Goclenius e con Clauberg prenderà il nome di ontologia, ma, dall'altro, difende una posizione che non è differente da quella di Suárez. Se, infatti, la terminologia contraddice quella di Suárez, sul piano concettuale le due posizioni sono per l'essenziale convergenti. Si comprende, allora, come la scelta cartesiana del termine di philosophia

Metaphysica generalis und eine Metaphysica specialis, «Zeitschrift für philosophische Forschung», XVI (1962), n. 2, p. 259-284.

37 Pereira, De Communibus rerum, I, 6, p. 23.

38 G. Crapulli, Mathesis Universalis. Genesi di un'idea nel XVI secolo, Roma, Edizioni dell'Ateneo, 1969. 
prima a scapito di metaphysica nasconda un disaccordo concettuale radicale con Pereira (e con Suárez).

Descartes non ha ripreso il nome utilizzato da Pereira se non per eliminare compiutamente la questione che voleva consacrarvi Pereira: la questione dell'ente in quanto ente ${ }^{39}$.

III.

L'importanza delle indagini di Marion, che non ho potuto riassumere qui se non a grandi tratti, risiede almeno a due livelli.

In primo luogo, direttamente, per la chiarificazione del contesto scolastico: la ricerca di Marion offre infatti una ricognizione pressoché esaustiva sulla nozione di metafisica in alcuni fra i principali autori della scolastica dell'epoca cartesiana. In secondo luogo, l'analisi storica di Marion risulta determinante anche indirettamente, ossia per l'interpretazione che, sulla base di essa, è offerta in rapporto al problema capitale dei rapporti fra Descartes e la storia della metafisica. In un contributo intitolato Descartes appartiene alla storia della metafisica?, il suo più vicino allievo, Vincent Carraud ha ribadito con argomenti di peso come l'indagine sul termine ante quem (come pure su quello post quem) sul concetto cartesiano di metafisica non abbia una funzione meramente storica, ma consenta di misurare precisamente un punto concettuale: ovverosia, precisamente, l'appartenenza di Descartes alla metafisica, lungamente data per scontata dalla storiografia tradizionale e messa finalmente in questione dalle indagini di Marion ${ }^{40}$.

Esiste, tuttavia, almeno un punto dell'analisi di Marion che mi sembra sollevare alcune difficoltà. Mi riferisco alla tesi secondo cui la scelta cartesiana del termine di filosofia prima sarebbe operata a

39 Marion 1986, p. 53.

40 V. Carraud, Descartes appartiene alla storia della metafisica?, in J.-R. Armogathe - G. Belgioioso, G. (a cura di), Descartes metafisico. Interpretazioni del Novecento, 1994, p. 165-177. 
detrimento di quello di metafisica ${ }^{41}$. Come si è visto, Marion basa una tale affermazione soprattutto su due testi, le due lettere di invio a Mersenne delle Meditationes, datate 11 novembre 1640. Ora, però, se si rileggono questi due testi, mi pare che essi non dicano precisamente ciò che Marion attribuisce ad essi, poiché attestano bensì una scelta a favore di filosofia prima, ma non anche che tale scelta sia operata a detrimento di 'metafisica'. Descartes si limita a sostenere che, poiché le Meditationes non trattano «pas seulement de Dieu et de l'Ame, mais en général de toutes les premières choses qu'on peut connaître en philosophant par ordre», allora esse si chiameranno di philosophia prima, ma non aggiunge che per questa - o altre ragioni - esse non dovranno chiamarsi di «metaphysica».

Il testo di Descartes, cioè, non presenta come esclusiva, anzi, neppure presenta, tout court, l'alternativa che Marion individua fra philosophia prima e metaphysica. La scelta cartesiana del termine di philosophia prima non è dunque, almeno testualmente, come invece sostiene Marion, «au détriment» di metaphysica.

Al contrario, resta possibile che Descartes abbia pensato le $\mathrm{Me}$ ditationes di 'filosofia prima' come delle meditazioni 'metafisiche' e che si sia limitato ad usare il primo termine non per escludere il secondo, ma per la semplice ragione che lo considerava, secondo una tradizione largamente attestata, come equivalente. L'ipotesi è confermata non solo dal fatto, che Marion pur rileva, che Descartes chiami a più riprese le Meditationes ' ma métaphysique '42, ma anche da un dato che le indagini di Marion non documentano: il fatto che il nome di Meditationes metaphysicæ sia stato utilizzato precisamente da Descartes per designare la sua opera. Lo attesta, incontrovertibilmente, un passo dell'articolo XXX della Prima pars dei Principia Philosophiæ:

\footnotetext{
$41 \quad$ Marion 1986, p. 45.

42 Marion 1986, p. 34, n. 34.
} 
Nec opus est ista pluribus verbis hoc in loco persequi, quoniam in Meditationibus Metaphysicis jam utcunque tractata sunt, et accuratior eorum explicatio ex sequentium cognitione dependet ${ }^{43}$.

Il nome di Meditazioni metafisiche, prima di essere il titolo dato dal duca De Luynes alla traduzione francese delle Meditationes nel 1647 (e di lì in poi avallato dagli editori successivi, a partire da Claude Clerselier e René Fedé), è una variante d'autore.

Mi pare dunque che i dati testuali non consentano in alcun modo di individuare nella scelta cartesiana del nome di 'philosophia prima' una scelta esclusiva del termine 'metafisica'. Certamente, questo pone il problema di un'esegesi alternativa, rispetto a quella proposta da Marion, delle due lettere a Mersenne sopra menzionate. Descartes vuole forse escludere un titolo quale Meditationes de Deo et de anima?

Si resta, qui, nel campo delle ipotesi. Quel che è certo, mi sembra, è che la ridefinizione cartesiana del concetto di filosofia prima, quale indagine sulle prime cose che si possono conoscere filosofando per ordine, e certamente per questo esclusiva di ogni indagine che abbia per oggetto l'ente (sia una regione particolare, sia una regione universale), non mi sembra necessariamente legata ad una scelta terminologica operata ad esclusione dell'utilizzazione del nome di metafisica.

Descartes ha visto davvero l'opposizione esclusiva che gli attribuisce Marion? O non potrebbe forse darsi, più semplicemente, che quella di Descartes segni, a un tempo, una ridefinizione del concetto di filosofia prima e di metafisica e che l'opzione a favore del termine di 'filosofia prima' costituisca l'espressione, più che di un'opzione a detrimento, di una sottodeterminazione teorica del concetto e del termine di metafisica?

Data de registro: 12/09/2016

Data de aceite: $26 / 10 / 2016$

43 AT VIII-1 17; B Op I 1732. 


\section{Note sul contributo di Jean-Luc Marion intorno al concetto di philosophia prima fra Descartes e la Scolastica*}

Riassunto: Questo articolo ha per oggetto l'indagine che Jean-Luc Marion ha consacrato nei suoi numerosi scritti e, in particolare, in Sur le prisme métaphysique de Descartes (1986), alla concezione cartesiana della metafisica in rapporto alla scolastica. Esso si propone di mostrare che le ricerche di Marion costituiscono uno dei maggiori contributi della letteratura critica cartesiana allo studio dei rapporti fra Descartes e la Scolastica. In particolare, esso discute e riconsidera la tesi di Marion secondo cui la scelta, da parte di Descartes, del termine di philosophia prima per intitolare le sue Meditationes sarebbe operata a detrimento del termine di metaphysica.

Parole chiave: Cartesio. Jean-Luc Marion. Scolastica. Metaphysica. Philosophia prima.

\section{Notas sobre a contribuição de Jean-Luc Marion acerca do estudo do conceito de philosophia prima em Descartes e na Escolástica}

* $\quad$ AT = René Descartes, Euvres, éd. par Ch. Adam et P. Tannery, nouv. présent. par J. Beaude, P. Costabel, A. Gabbey et B. Rochot, 11 vols., Paris, Vrin, 19641974 ; B = René Descartes, Tutte le lettere 1619-1650 (2005), a cura di G. Belgioioso, con la collaborazione di I. Agostini, F. Marrone, F. A. Meschini, M. Savini e di J.-R. Armogathe, Bompiani, Milano, 2009; B Op I = René Descartes. Opere 1637-1649, a cura di G. Belgioioso, con la collaborazione di I. Agostini, F. Marrone, M. Savini, Bompiani, Milano, 2009; DM = F. Suárez, Disputationes metaphysicae, in Opera Omnia, 28 voll., Parisiis, apud Ludovicum Vivès, 1856-1878, voll. XXV-XXVI; Marion 1986 = Sur le prisme métaphysique de Descartes. Constitution et limites de l'onto-théo-logie dans la pensèe cartésienne, Paris, PUF, 1986.

** Professor de Filosofia da Universidade do Salento (Lecce). E-mail: igor.agostini@unisalento.it 
Resumo: Este artigo tem por objeto a indagação que Jean-Luc Marion consagrou nos seus numerosos escritos e, em particular, em Sur le prisme métaphysique de Descartes (1986), à concepção cartesiana da Metafísica em relação à Escolástica. Propõem-se mostrar que as pesquisas de Marion constituem uma das maiores contribuições da literatura crítica cartesiana ao estudo das relações entre Descartes e a Escolástica. Em particular, o artigo discute e reconsidera a tese de Marion segundo a qual a escolha, da parte de Descartes, do termo philosophia prima para intitular as suas Meditationes teria sido feita em detrimento do termo metaphysica.

Palavras-chave: Descartes. Jean-Luc Marion. Escolástica. Metaphysica. Philosophia prima.

\title{
Notes on Jean-Luc Marion's contribution on the study of the notion of prima philosophia in Descartes and the scholasticism
}

\begin{abstract}
This article addresses the enquiry devoted by Jean-Luc Marion in his many works and, in particular, in Sur le prisme métaphysique de Descartes (1986), to Descartes's conception of Metaphysics considered in connection to scholasticism. The aim is to demonstrate that Marion's researches constitue in Cartesian scholarship one of the main contributions to the study of the relationships between Descartes and Scholasticism. At the same time, the article discusses and reconsiders Marion's claim that Descartes's choice of the term philosophia prima for the title if his Meditationes would go to the detriment of the term metaphysica.
\end{abstract}

Keywords: Descartes. Jean-Luc Marion. scholasticism. Metaphysica. Philosophia prima.

I.

Le principali opere interpretative consacrate da Jean-Luc Marion a Descartes, ristampate a più riprese e in alcuni casi tradotte in più lingue, sono ben note: la fondamentale trilogia su L'ontologie grise (1975, II ed. rev. 1981), La théologie blanche (1981, II ed. rev. 1986) et Le prisme métaphysique (1986), un cospicuo numero di articoli di grande densità teorica, per lo più raccolto nei due volumi delle Questions cartésiennes 
(1991 e 1996) e, infine, l'ultima monografia su La pensée passive chez Descartes (2013) ${ }^{1}$.

Questi lavori offrono un contributo interpretativo allo studio di Descartes che, con ogni probabilità, è il maggiore dell'ultimo cinquantennio, dopo la grande generazione degli Alquié, Gouhier, Gueroult, Laporte. Non si tratta di un'opinione personale, ma di un dato oggettivo, attestato dalla larga diffusione delle tesi di Marion, che in Francia, e non solo, costituiscono spesso il punto di partenza di numerosi studi che si propongono in molti casi proprio di ridiscutere le stesse tesi interpretative marioniane.

Anzitutto due, sono gli aspetti del contributo di Marion che hanno suscitato l'attenzione degli studiosi. In primo luogo, la sua lettura interna dei testi cartesiani, ossia il suo apporto sul piano esegetico di questioni fondamentali quali la teoria della creazione delle verità eterne, il concetto cartesiano di metafisica, l'io, Dio, qui solo menzionate che alcune tematiche di particolare interesse. In secondo luogo, l'intenso confronto con la lettura di grandi filosofi quali, soprattutto, Husserl ed Heidegger: basti pensare, su quest'ultimo punto, al tentativo di lettura di Descartes alla luce del paradigma onto-teologico della metafisica realizzato nel Prisme e già abbozzato nella Théologie blanche, ma anche i contributi de La pensée passive verso l'individuazione dell'emergenza della chair rispetto al corpo fisico.

C'è però un altro aspetto della lettura di Marion, peraltro strettamente legato a quello interpretativo (che su di esso si appoggia), a mio parere, altrettanto importante, ossia il suo contributo ai rapporti fra Descartes e la Scolastica: credo si possa infatti affermare che in tale direzione il contributo di Marion sia senz'altro, dopo quello di Etienne Gilson, uno

Sur la théologie blanche de Descartes. Analogie, création des vérités éternelles et fondement (1981), PUF, Paris, 1991²; Descartes et l'onto-théologie, in «Bulletin de la Société française de philosophie», LXXVI (1982), pp. 117-171; Marion 1986; Questions cartésiennes. Méthode et métaphysique, Paris, PUF, 1991; Questions cartésiennes II. Sur l'ego et sur Dieu, PUF, Paris, 1996; Sur la pensée passive de Descartes, Paris, PUF, 2013. 
dei maggiori del secolo scorso, insieme a quello di autori quali Pierre Garin, Timothy J. Cronin e, Norman J. Wells.

Il suo fondamentale apporto si estende difatti, limitandomi al nucleo essenziale, a quattro questioni: la questione dell'analogia; la questione della metafisica; la questione dei nomi divini; la questione della sostanza. Si tratta di tematiche capitali che in questa sede, per ragioni di spazio, non mi è possibile ripercorrere a dovere. Mi soffermerò, infatti, solo su una di esse, sia per la sua importanza, sia perché, forse, a differenza delle altre, ha suscitato meno attenzione all'interno del dibattito storiografico successivo.

Mi riferisco, in particolare, alla questione della metafisica, oggetto, peraltro, del primo capitolo de Le prisme métaphysique de Descartes.

\section{II.}

Il punto di partenza dell'indagine di Marion sul concetto cartesiano di metafisica è costituito dalla stessa accezione del termine che si ritrova, in Descartes, sin dalle lettere del 1630 a Mersenne sulle verità eterne:

Pensai-je avoir trouvé comment on peut démontrer les vérités métaphysiques, d'une façon qui est plus évidente que les démonstrations de Géométrie ${ }^{2}$.

Poiché la metafisica è qui intesa come scienza che sorpassa le altre scienze («Dieu est une cause dont la puissance surpasse les bornes de l'entendement humain $»^{3}$ ), Marion vi ritrova il concetto aristotelico di philosophia prima, o meglio, l'etimologia della philosophia prima quale scienza che si eleva, in quanto prima, al di sopra delle altre scienze teoriche.

Si tratta di un'etimologia largamente diffusa nella scolastica degli anni di Descartes, come lo stesso Marion dimostra attraverso il reperimento di numerosi testi:

AT I 144; B 30, p. 144.

AT I 150; B 31, p. 150. 
[...] Hinc factum est; ut scientia ipsa, quæ his libris traditur, simplici nomine appellari soleat a Recentioribus Metaphysica, quasi postnaturalium aut Supranaturalium scientia, cum ab Aristotele sæpe appellatur prima philosophia ${ }^{4}$.

[...] Ex quo etiam metaphysica nominata est; quod nomen non tam $\mathrm{ab}$ Aristotele, quam ab ejus interpretatibus habuit; sumptum vero est ex inscriptione quam Aristoteles suis Metaphysicæ libris præscripsit,

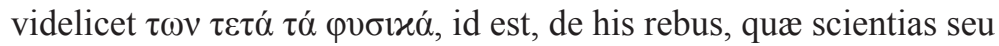
res naturales consequuntur ${ }^{5}$.

[...] hanc scientiam transphysicam seu postphysicam vocavit [sc. Aristotele] $]^{6}$.

[...] quia Physicam ordine doctrinæ sequitur, sive quia illam prætergreditur?

[...] Tandem appellatur Metaphysica, id est post-naturalis scientia, seu disciplina rerum res naturales consequentium ${ }^{8}$.

Difatti Descartes, in linea con questa tradizione, scrive: «La métaphysique se définit et se constitue par l'acte même de transgresser toute autre science» ${ }^{9}$. Questo non solo nel 1630, ma anche nel 1647, nella Lettre-Préface dei Principes de la philosophie, quando la metafisica

4 P. Fonseca, Commentarii in libros metaphysicorum Aristotelis Stagiritae, 4 voll., Coloniæ, Sumptibus Lazari Zetzneri, 1615-1619, rist. anast. : Hildesheim, G. Olms, 1964 (= Metaphysica), Proem., 8, vol. I p. 35, cit. in Marion 1986, p. 24, n. 20.

5 DM, I, 1, Proem., ed. Vivès XXV 2b, cit. in Marion 1986, pp. 24-25, n. 21.

6 DM, I, 4, 13, ed. Leon. XXV 29a, cit. in Marion 1986, pp. 24-25, n. 21.

7 Eustachius a S. Paulo, Summa philosophiae quadripartita, Parigi, apud Carolum Chastellain 1609 (= Summa philosophice), Metaphysica, Praefatio, 1, p. 1., cit. in Marion 1986, p. 25 nota 22 .

8 Abra de Raconis, Summa totius philosophice : id est, logica, ethica, physica, metaphysica et horum omnium Compendium, Coloniæ Agrippinæ, ex officina Choliniana, suptibus Petri Cholini, 1629 (= Summa totius philosophice), Metaphysica, Præfatio, p. 1., cit. in Marion 1986, p. 25, n. 22.

9 Marion 1986, p. 25. 
cartesiana ha ormai acquisito una fisionomia ben definita: «[...] la vraie Philosophie, dont la première partie est la Métaphysique» ${ }^{10}$.

Inoltre, il debito di Descartes nei confronti della tradizione scolastica si rileva, secondo Marion, anche in un secondo carattere che l'autore delle Meditationes attribuisce alla metafisica: l'astrazione dalle condizioni sensibili. La metafisica comincia difatti per Descartes allorché la mente si ritrae dai sensi, indirizzandosi verso le «res intelligibiles tantum, atque $\mathrm{ab}$ omni materia secretas ${ }^{11}$.

È, questo, un altro tratto tipico della metafisica secondo i maggiori autori dell'epoca di Descartes rilevato da Marion, ancora una volta, attraverso numerose citazioni tratte dai principali testi scolastici del tempo:

Tertia est abstractio rei ab omni materia, tam secundum rem, quam secundum rationem, quam abstractionem cernimus in Deo et intelligentiis $[\ldots]^{12}$.

Abstrahit enim hæc scientia de sensibilibus seu materialibus rebus (quæ physice dicuntur, quoniam in eis naturalis philosophia versatur) et res divinas et materia separatas et communes rationes entis, quæ absque materia existere possunt, contemplatur: et ideo metaphysica dicta est, quasi post physicam seu ultra physicam constituta ${ }^{13}$.

[...] metaphysicam esse scientiam quæ ens, in quantum ens, seu in quantum a materia abstrahit secundum esse, contemplatur ${ }^{14}$.

[...] ens abstractum a Deo et creaturis statuendum est adæquatum metaphysicæ objectum ${ }^{15}$.

$10 \quad$ AT IX-2 14; B Op I 2228.

11 AT VII 53, B Op I 750.

12 Pererius, De Communibus omnium rerum naturalium principiis et affectionibus (1576), Parisiis : apud Micaelem Sonnium, via Iacobea, sub scuto Basiliensi, 1579 (= De Communibus), I, 5, p. 16, ed. 1579, cit. in Marion 1986, p. 28, n. 26.

13 Suárez, DM, I, Proemium, ed. Vivès XXV 2a, in Marion 1986, p. 28, n. 26.

14 Suárez, DM, I, 3, 1, ed. Vivès, XXV 22b, in Marion 1986, p. 28, n. 26.

15 Abra de Raconis, Summa totius philosophice, Metaphysica, Præludium quartum, 2, prop. post., p. 4, in Marion 1986, p. 28, n. 26. 
Ex his colligi potest hæc Metaphysicæ definitio ut videlicet dicatur scientia theorica de ente reali per se et completo ab omni materia saltem secundum indifferentiam abstracto ${ }^{16}$.

Certamente, Descartes allarga in una direzione sconosciuta agli autori scolastici questa nozione di metafisica: essa non astrae solo dalle cose sensibili, ma si spinge sino ad abducere mentem a sensibus ${ }^{17}$. Ma questa estensione, lungi dall'attenuare la continuità della concezione cartesiana della metafisica con quella scolastica, la rinforza:

Les occurrences cartésiennes de 'métaphysique', quelque rares et éparses qu'elles soient, retrouvent donc une réelle cohérence sitôt qu'elles sont rapportées aux déterminations aristotéliciennes, mais aussi scolaires, admises par les contemporains de Descartes. Ainsi, dès 1630, Descartes reprend-il à son compte l'ambition d'Aristote, en E1, de constituer une science outrepassant la physique et les mathématiques; il reprend aussi l'abstraction comme l'acte privilégié pour transgresser les deux sciences secondes, et il en élargit la puissance par l'abductio mentis a sensibus ${ }^{18}$.

Questo dato non deve essere in alcun modo trascurato: basti pensare che, nell'Index scolastico-cartésien di Gilson, la voce metafisica costituisce solo una piccola sottovoce (solo quattro testi) della voce 'Fi-

16 Eustachius a S. Paulo, Summa philosophice, Metaphysica, Prcefatio, 2, p. 5, in Marion 1986, p. 29, n. 27.

17 Synopsis, B Op I 695 (AT VII 12, 11. 7-8); Meditationes, IV, B Op I 751 (AT VII 52, 11. 23-24); A Mersenne, 20 aprile 1637, B 104, p. 367 (AT I 350, 1. 29-351, 1. 2); A Vatier, 22 febbraio 1638, B 149, p. 549 (AT I 560, 11. 13-27). Cfr. anche Discours, IV, B Op I 67 (AT VII 37 11. 1-5) e, l'aggiunta: «ex eo est quod nunquam animum a sensibus abducant, et supra res corporeas attollant» in R. Descartes, Specimina Philosophiae seu: Dissertatio de Methodo recte regendae rationis, et veritatis in scientiis investigandae: Dioptrice, et Meteora. Ex gallico translata et ab auctore perlecta, variisque in locis emendata, Amstelodami, apud Ludovicum Elzevirium, 1644, rist. anast.: Lecce, Conte, 1998, a cura di J.-R. Armogathe e G. Belgioioso, p. 34.

18 Marion 1986, p. 33. 
losofia' ${ }^{19}$. Ma è proprio in tal modo che si genera la questione decisiva, secondo Marion: una volta dimostrato, cioè, che il termine ed il concetto di metafisica intervengono nel corpus cartesiano, occorre difatti chiarire quale sia il concetto propriamente cartesiano di metafisica:

Sur ce sol désormais assuré peut s'énoncer enfin la question décisive: puisque intervient bien «métaphysique» dans le corpus cartésien, comment s'y trouve proprement compris le concept de métaphysique? ${ }^{20}$

Il che significa misurare, precisamente «grâce a ces similitudes, les dissimilitudes qui provoquent les décisions proprement cartésiennes», ovverosia «s'interroger sur l'identité de la métaphysique instaurée en 1641 par les Meditationes» ${ }^{21}$. Ora, secondo Marion:

Or, c'est précisément ici que le déplacement s'accomplit le plus indiscutablement, puisque Descartes dénie expressement aux Meditationes le titre de metaphysicæ. L'originalité des Meditationes tient à ceci, qu'elles ne sont pas metaphysicæ' ${ }^{22}$.

Si tratta, sostiene Marion, di un fatto mascherato dalla traduzione francese delle Meditationes, che De Luynes rende appunto Méditations métaphysiques, ma anche, almeno in parte, da Descartes stesso, che, come avremo modo di ripetere più avanti, presenta a più riprese le Meditationes come «ma métaphysique». Tuttavia, è Descartes stesso che, per due volte, espone con nettezza i motivi che lo interdicono nel qualificare le Meditationes come «metaphysicæ». Questo, nelle

19 Cfr. E. Gilson, Index scolastico-cartésien. Seconde édition revue et augmentée seule autorisée par l'auteur (1913), Paris, Vrin, 1979, textes nn. 358-361. Nell'aggiornamento dell'opera di Gilson condotto nel Nouvel Index scolastico-cartésien, a cura del sottoscritto, con la collaborazione di S. Agostini, C. Catalano e I. Coluccia, la voce «metaphysica» tiene largamente conto della documentazione testuale offerta da Marion.

$20 \quad$ Marion 1986, p. 33.

21 Marion 1986, p. 34.

22 Marion 1986, p. 34. 
due lettere di invio a Mersenne delle Meditationes, datate entrambe 11 novembre 1640 :

J'ai envoyé dès hier ma Métaphysique à $\mathrm{M}$. de Zuylichem pour vous l'adresser; mais il ne l'enverra que dans huit jours, car je lui ai donné ce temps pour la voir. Je n'y ai point mis de titre, mais il me semble que le plus propre sera de mettre Renati Descartes Meditationes de prima Philosophia; car je ne traite point en particulier de Dieu et de l'Ame, mais en général de toutes les premières choses qu'on peut connaître en philosophant ${ }^{23}$.

Je vous envoie enfin mon écrit de Métaphysique, auquel je n'ai point mis de titre, afin de vous en faire le parrain, et vous laisser la puissance de le baptiser. Je crois qu'on le pourra nommer, ainsi que je vous ai écrit par ma précédente, Meditationes de prima Philosophia; car je n'y traite pas seulement de Dieu et de l'Ame, mais en général de toutes les premières choses qu'on peut connaître en philosophant par ordre ${ }^{24}$.

Secondo l'ipotesi di Marion, questi testi attesterebbero in maniera inequivocabile una decisione terminologica costante, da parte di Descartes, che è, a un tempo, a favore di philosophia prima, e a detrimento $d i$ metaphysica:

Il s'agit donc d'une decision nette et constante, au detriment de métaphysique/metaphysica, et en faveur de la philosophie premièrel prima philosophia, au sens strict de la filosof.... a prèth et suivant la nomenclature d'Aristote ${ }^{25}$.

Questa scelta lessicale induce una questione senza rispondere alla quale la scelta di Descartes, per quanto netta, resterebbe insignificante: perché una tale scelta, appunto, da parte di Descartes? Secondo l'ipotesi

23 AT III 235; B 284, p. 1324.

24 AT III 240; B 285, p. 1328.

25 Marion 1986, p. 35-36. 
di Marion, il fatto è che Dio e l'anima esauriscono, in Descartes, il campo della metafisica e, conseguentemente, si richiede una nuova disciplina che abbia per oggetto, essa sola, tutte le prime cose, quali esse siano:

Puisque Dieu et l'âme épuisent pour Descartes le champ de la métaphysique, l'élargissement de ce champ postule aussitôt une nouvelle discipline - la philosophie première, qui porte, elle seule, sur toutes les premières choses, quelles qu'elles soient et quelle qu'en soit la primauté26.

In questo modo:

[...] la philosophie première outrepasse la métaphysique en s'étendant à toute primauté; ainsi est-elle universelle en tant que première ${ }^{27}$.

La primauté in questione è quella nell'ordine della conoscenza. Il punto è capitale, in quanto determina una reinterpretazione della stessa primauté che viene staccata da ogni ambito ontologico (sia di Dio e dell'anima, sia dell'ente in generale) e collocata puramente sull'ordine della conoscenza: di conseguenza, la filosofia prima non desumerà la sua primauté da determinate regioni dell'ente supposte intrinsecamente prime (Dio, l'anima, le intelligenze separate), né dall'ente in quanto tale, poiché, una volta entrato in scena l'ordo cognoscendi, l'ente non entra più in gioco.

Una tale concezione della metafisica è ratificata dalla definizione della Lettre-Préface ai Principes:

[...] La vrai philosophie dont la première partie est la Métaphysique, qui contient les principes de la connaissance ${ }^{28}$.

26 Marion 1986, p. 37.

27 Marion 1986, p. 38.

28 AT IX-2 14; B Op I 2228. 
Definizione capitale, che, via Clauberg, giungerà sino a Wolff, e sarà assunta in proprio anche da Kant. Così, lungi dal rimettere in questione il primato della filosofia prima, la scelta terminologica di 'metafisica' la conferma, alla luce del nuovo concetto di primauté. In questo modo, «Descartes pense la métaphysique comme philosophie première, parce qu'il modifie de fond en comble l'essence de la primauté» ${ }^{29}$.

Per comprendere l'importanza di una tale decisione è necessario misurarne la portata concettuale e, a tal fine, rapportare la definizione cartesiana di filosofia alle definizioni precedenti, sia di metafisica, sia di filosofia prima.

È qui che l'analisi marioniana del background scolastico della filosofia di Descartes si fa più intensa. Il paragrafo 4 del capitolo I del Prisme contiene un contributo decisivo alla ricostruzione delle differenti definizioni di metafisica e di filosofia prima della scolastica dell'età moderna, del tutto assente nell'ambito della storiografia cartesiana, ivi compreso l'Index scolastico-cartésien di Gilson.

Dalle analisi di Marion risulta con evidenza che, nei contemporanei di Descartes, è largamente diffusa una tesi: la metaphysica ha per oggetto sia gli enti più nobili, sia l'ente in quanto ente. Le due scienze che in Aristotele sono distinte, o, comunque, non riunite sotto un titolo comune, ossia la philosofia prima che ha per oggetto gli enti più divini, e 'quella scienza' che ha per oggetto l'ente in quanto ente, sono senz'altro unificate sotto l'unico nome di 'metafisica'.

Così, in Suárez:

[...] Hæc scientia [...] tractat [...] de nobilissimis entibus et de universalissimis rationibus entis et maxime abstractis ${ }^{30}$.

Nell'affermare, così esplicitamente, l'unità specifica della metafisica, Suárez utilizza però, nondimeno, una distinzione terminologica:

29 Marion 1986, p. 43.

30 Suárez, DM, I, 4, 2, ed. Vivès XXV 26b, cit. in Marion 1986, p. 43, n. 42. 
Nihilominus asserendum est cum communi sententia, metaphysicam simpliciter esse unam scientiam specie. Hæc enim videtur clara mens Aristotelis in toto prooemio, seu c. 1 et 2, lib. I Metaph., ubi semper de hac scientia tamquam de una specie loquitur, eique tamquam uni et eidem attribuit nomina et attributa, quæ partim illi conveniunt, secundum quod versatur circa Deum et intelligentias; sic enim vocatur theologia, seu scientia divina, et prima philosophia; partim ut versatur circa ens in quantum ens, et prima attributa, et principia eius, qua ratione dicitur scientia universalis et metaphysica. Sapientia autem vocatur, quatenus hæc omnia complectitur, et prima principia, primasque rerum causas contemplatur ${ }^{31}$.

In breve, posta l'unità specifica della metafisica quale scienza, per Suárez la filosofia prima (in questo, sinonimo di teologia e scienza divina) indica la scienza che ha per oggetto Dio e le intelligenze; la metafisica (in questo, sinonimo di scienza universale) indica la scienza che ha per oggetto l'ente in quanto ente. È proprio qui che si situa la differenza capitale fra Descartes e Suárez. Infatti, se per Suárez la philosophia prima non concerne che Dio e le intelligenze, per Descartes è la metafisica ad avere per oggetto gli enti più nobili (Dio e l'anima), mentre la filosofia prima ha per oggetto le prime cose che possono conoscersi filosofando con ordine.

In tal modo, la metafisica, che per Suárez copre un dominio universale dell'ente (l'ente in quanto ente), per Descartes investe un dominio più particolare (Dio e l'anima); e la filosofia 'prima', che per Suárez copre un dominio particolare dell'ente (Dio e le intelligenze), per Descartes investe un dominio più in generale. In sostanza:

Tout se passe comme si Descartes ne reprenait le couple suárezien de prima philosophia/metaphysica que pour en inverser les caractéristiques: la première devient universelle et non théologique, la seconde devient particulière et théologique ${ }^{32}$.

31 Suárez, DM I, 3, 9, ed. Vivès XXV 24b.

32 Marion 1986, p. 46. 
Ad essere ridefinita, qui, è la primauté della filosofia prima, stabilita non dall'ordine dell'essere, ma dalla conoscenza e, insieme, dall'universalità. Un tale ribaltamento, secondo Marion, merita tanto più attenzione quanto più contraddice l'uso dei contemporanei di Descartes. Oltre che in Suárez, infatti, è largamente attestata nella gran parte degli autori scolastici dell'epoca anche l'identificazione dell'oggetto della filosofia prima agli enti più nobili e dell'oggetto della metafisica all'ente in quanto ente. Alcuni degli esempi addotti da Marion lo attestano in modo cristallino:

Dissentiunt de re propositia Philosophi; alii enim Deum, alii substantias separatas, alii substantiam in communi, alii ens finitum quod vocant prædicamentale, objectum esse Metaphysicæ contendunt: sed hi omnes non satis amplum illud assignant, ut patebit. [...] Est igitur longe verisimilior communis sententia: Objectum per se et adæquatum Metaphysicæ (de eo enim, non vero de objecto per accidens, aut partiali proposita quæstio intelligenda est) esse ens reale, per se, completum, commune Deo et rebus creatis [... $]^{33}$.

[...] hæc Metaphysicæ definitio, ut videlicet dicatur theoretica de ente reali per se et completo ab omni materia saltem secundum indifferentiam abstracto ${ }^{34}$.

$[\ldots]$ nomine primæ philosophiæ $[\ldots]$ cum de præstantissimis rebus, Deo nempe et angelis, verba faciat ${ }^{35}$.

La posizione di Descartes non è, tuttavia, senza antecedenti. C'è infatti un autore ${ }^{36}$ che aveva invece inteso la filosofia prima come

33 Eustachius a S. Paulo, Summa philosophice, Metaphysica, Præfatio, 2, p. 3, cit. in Marion 1986, p. 47, n. 46.

34 Eustachius a S. Paulo, Summa philosophice, Metaphysica, Præfatio, 2, p. 5, cit. in Marion 1986, p. 47, n. 46.

35 Abra de Raconis, Summa totius philosophice, Metaphysica, Præfatio, p. 1, cit. in Marion 1986, p. 46, n. 44.

36 La cui originalità era stata richiamata già da E. Vollrath, cui Marion 1986, p. 46, n. 9, si richiama esplicitamente: cfr. E. Vollrath, Die Gliederung der Metaphysik in eine 
universale: Benito Pereira, nel De communibus omnium rerum naturalium principiis et affectionibus libri quindecim (1576). Qui, infatti, senz'altro, Pereira sostiene che l'oggetto della filosofia prima sono le proprietà trascendentali ed universali, mentre l'oggetto della metafisica sono le intelligenze:

[...] Necesse est duas scientias distinctas inter se. Unam quæ agat de trascendentibus et universalissimis rebus. Alteram quæ de intelligentiis. Illa dicitur prima Philosophia et scientia universalis, hæc vocabitur proprie Metaphysica, Theologia, sapientia, divina scientia $[\ldots]^{37}$.

La tesi di una possibile influenza di Pereira su Descartes su questo punto è sostanziata dal fatto che, come dimostrato da Giovanni Crapulli, è noto che Descartes conoscesse lo stesso, poiché è da lui che desume il sintagma di Mathesis universalis ${ }^{38}$. Ma, al di là dell'effettiva influenza storica, Marion insiste sulla convergenza tematica: la primauté della filosofia prima resta definita non dalla nobiltà del suo oggetto, ma dalla sua universalità. Tuttavia, secondo Marion, la convergenza di Descartes con Pereira è solo molto parziale, puramente nominale. Per Pereira, difatti, la filosofia prima ha per oggetto le determinazioni trascendentali dell'ente, dunque, in ultima istanza, l'ente in quanto ente. La sua universalità, pertanto, dipende dall'universalità delle determinazioni dell'ente stesso. In questo modo, da un lato, Pereira anticipa la scienza che dopo Goclenius e con Clauberg prenderà il nome di ontologia, ma, dall'altro, difende una posizione che non è differente da quella di Suárez. Se, infatti, la terminologia contraddice quella di Suárez, sul piano concettuale le due posizioni sono per l'essenziale convergenti. Si comprende, allora, come la scelta cartesiana del termine di philosophia

Metaphysica generalis und eine Metaphysica specialis, «Zeitschrift für philosophische Forschung», XVI (1962), n. 2, p. 259-284.

37 Pereira, De Communibus rerum, I, 6, p. 23.

38 G. Crapulli, Mathesis Universalis. Genesi di un'idea nel XVI secolo, Roma, Edizioni dell'Ateneo, 1969. 
prima a scapito di metaphysica nasconda un disaccordo concettuale radicale con Pereira (e con Suárez).

Descartes non ha ripreso il nome utilizzato da Pereira se non per eliminare compiutamente la questione che voleva consacrarvi Pereira: la questione dell'ente in quanto ente ${ }^{39}$.

III.

L'importanza delle indagini di Marion, che non ho potuto riassumere qui se non a grandi tratti, risiede almeno a due livelli.

In primo luogo, direttamente, per la chiarificazione del contesto scolastico: la ricerca di Marion offre infatti una ricognizione pressoché esaustiva sulla nozione di metafisica in alcuni fra i principali autori della scolastica dell'epoca cartesiana. In secondo luogo, l'analisi storica di Marion risulta determinante anche indirettamente, ossia per l'interpretazione che, sulla base di essa, è offerta in rapporto al problema capitale dei rapporti fra Descartes e la storia della metafisica. In un contributo intitolato Descartes appartiene alla storia della metafisica?, il suo più vicino allievo, Vincent Carraud ha ribadito con argomenti di peso come l'indagine sul termine ante quem (come pure su quello post quem) sul concetto cartesiano di metafisica non abbia una funzione meramente storica, ma consenta di misurare precisamente un punto concettuale: ovverosia, precisamente, l'appartenenza di Descartes alla metafisica, lungamente data per scontata dalla storiografia tradizionale e messa finalmente in questione dalle indagini di Marion ${ }^{40}$.

Esiste, tuttavia, almeno un punto dell'analisi di Marion che mi sembra sollevare alcune difficoltà. Mi riferisco alla tesi secondo cui la scelta cartesiana del termine di filosofia prima sarebbe operata a

39 Marion 1986, p. 53.

40 V. Carraud, Descartes appartiene alla storia della metafisica?, in J.-R. Armogathe - G. Belgioioso, G. (a cura di), Descartes metafisico. Interpretazioni del Novecento, 1994, p. 165-177. 
detrimento di quello di metafisica ${ }^{41}$. Come si è visto, Marion basa una tale affermazione soprattutto su due testi, le due lettere di invio a Mersenne delle Meditationes, datate 11 novembre 1640. Ora, però, se si rileggono questi due testi, mi pare che essi non dicano precisamente ciò che Marion attribuisce ad essi, poiché attestano bensì una scelta a favore di filosofia prima, ma non anche che tale scelta sia operata a detrimento di 'metafisica'. Descartes si limita a sostenere che, poiché le Meditationes non trattano «pas seulement de Dieu et de l'Ame, mais en général de toutes les premières choses qu'on peut connaître en philosophant par ordre», allora esse si chiameranno di philosophia prima, ma non aggiunge che per questa - o altre ragioni - esse non dovranno chiamarsi di «metaphysica».

Il testo di Descartes, cioè, non presenta come esclusiva, anzi, neppure presenta, tout court, l'alternativa che Marion individua fra philosophia prima e metaphysica. La scelta cartesiana del termine di philosophia prima non è dunque, almeno testualmente, come invece sostiene Marion, «au détriment» di metaphysica.

Al contrario, resta possibile che Descartes abbia pensato le $\mathrm{Me}$ ditationes di 'filosofia prima' come delle meditazioni 'metafisiche' e che si sia limitato ad usare il primo termine non per escludere il secondo, ma per la semplice ragione che lo considerava, secondo una tradizione largamente attestata, come equivalente. L'ipotesi è confermata non solo dal fatto, che Marion pur rileva, che Descartes chiami a più riprese le Meditationes ' ma métaphysique '42, ma anche da un dato che le indagini di Marion non documentano: il fatto che il nome di Meditationes metaphysicæ sia stato utilizzato precisamente da Descartes per designare la sua opera. Lo attesta, incontrovertibilmente, un passo dell'articolo XXX della Prima pars dei Principia Philosophiæ:

\footnotetext{
$41 \quad$ Marion 1986, p. 45.

42 Marion 1986, p. 34, n. 34.
} 
Nec opus est ista pluribus verbis hoc in loco persequi, quoniam in Meditationibus Metaphysicis jam utcunque tractata sunt, et accuratior eorum explicatio ex sequentium cognitione dependet ${ }^{43}$.

Il nome di Meditazioni metafisiche, prima di essere il titolo dato dal duca De Luynes alla traduzione francese delle Meditationes nel 1647 (e di lì in poi avallato dagli editori successivi, a partire da Claude Clerselier e René Fedé), è una variante d'autore.

Mi pare dunque che i dati testuali non consentano in alcun modo di individuare nella scelta cartesiana del nome di 'philosophia prima' una scelta esclusiva del termine 'metafisica'. Certamente, questo pone il problema di un'esegesi alternativa, rispetto a quella proposta da Marion, delle due lettere a Mersenne sopra menzionate. Descartes vuole forse escludere un titolo quale Meditationes de Deo et de anima?

Si resta, qui, nel campo delle ipotesi. Quel che è certo, mi sembra, è che la ridefinizione cartesiana del concetto di filosofia prima, quale indagine sulle prime cose che si possono conoscere filosofando per ordine, e certamente per questo esclusiva di ogni indagine che abbia per oggetto l'ente (sia una regione particolare, sia una regione universale), non mi sembra necessariamente legata ad una scelta terminologica operata ad esclusione dell'utilizzazione del nome di metafisica.

Descartes ha visto davvero l'opposizione esclusiva che gli attribuisce Marion? O non potrebbe forse darsi, più semplicemente, che quella di Descartes segni, a un tempo, una ridefinizione del concetto di filosofia prima e di metafisica e che l'opzione a favore del termine di 'filosofia prima' costituisca l'espressione, più che di un'opzione a detrimento, di una sottodeterminazione teorica del concetto e del termine di metafisica?

Data de registro: 12/09/2016

Data de aceite: $26 / 10 / 2016$

43 AT VIII-1 17; B Op I 1732. 